\title{
Synthesis of Barbituric and Thiobarbituric Acids Bearing 5,6-Diphenyl-1,2,4-Triazin-3-yl Moiety as CDK2 Inhibitors of Tumor Cells
}

\author{
Dina Abed Bakhotmah \\ Department of Chemistry, Faculty of Science, King Abdulaziz University, Jeddah, Saudi Arabia
}

Email address:

dbakhotmah@kau.edu.sa,d.bakhotmah@yahoo.com

\section{To cite this article:}

Dina Abed Bakhotmah. Synthesis of Barbituric and Thiobarbituric Acids Bearing 5,6-Diphenyl-1,2,4-Triazin-3-yl Moiety as CDK2 Inhibitors of Tumor Cells. American Journal of Heterocyclic Chemistry. Vol. 5, No. 4, 2019, pp. 76-80. doi: 10.11648/j.ajhc.20190504.11

Received: August 27, 2019; Accepted: September 10, 2019; Published: October 11, 2019

\begin{abstract}
Synthesis of several new diphenyl-1',2',4'-triazin-3'-yl barbituric acid are described. The method involves addition reaction of isocyanate and isothiocyanate and 3-amino-5,6-diphenyl-1,2,4-triazine (1) to give $\mathrm{N}^{1}, \mathrm{~N}^{3}$-disubstituted urea 2 and $\mathrm{N}^{1}, \mathrm{~N}^{3}$-disubstituted thioureas 3 and 4 respectively. Further, ring closure reactions with malonate ester give barbituric acid 5 and thiobarbituric acid 6 and 7. The Presence of the active methylene in the skeleton of compound 5-7 at C-5 are deduced by condensation with pyridine-4-carboxyladehyde to give barbituric and thiobarbituric acids (8-10). Further fluoroacylation of compounds 5-7, afforded 1-(cyclohexyl/methyl/phenyl)-3-(5',6'-diphenyl-1',2',4'-triazin-3'-yl)-5-(trifluoracetyl)-5Hbarbituric/thiobarbituric acids (11-13). Synthesis compounds of the series 5-(trifluoroacetyl) barbituric acid (11) and 5(trifluoroacetyl) thiobarbituric acids (12 and 13) were able to inhibit activity of CDK2 in a biochemical assay with IC50 values comparable to olomoucine. In addition, a pyridine side chain at C-5 (compound 9 and 10) significantly decreases CDK2 inhibitory activity.
\end{abstract}

Keywords: Barbituric, Thiobarbituric, 1,2,4-triazine, $\mathrm{CDK}_{2}$, Malonate Ester, Trifluoroacylation

\section{Introduction}

The success of Imatinib mesylate as the first small molecule targeted kinase inhibitor for use in cancer therapy [1], are validated protein kinases as important drug targets in the treatment of human diseases [2]. The protein kinase family constitutes the largest gene-family for therapeutic development, and hence there is an urgent need to develop and discover compounds that can both serve as pharmacological probes and lead compounds for further drug development [3]. The condensed and substituted thiobarbituric acids possesses diverse pharmacological profile such as antimicrobial, selective cell adhesion inhibitors and DNA cleavage activities [4]. Additionally Barbiturate and thiobarbiturate derivatives attracted considerable attention owing to their various biological effects such as inhibiting collagenase-3 (MMP-3), recombinant cytochrome $\mathrm{P} 450$ enzymes and antiinflammatory analgesic [5]. In focused the Polyfunctional heterocyclic nitrogen systems are essential for drug discovery
[6], For example, pyrazolyl-1,2,4-triazines [5], 3functionalized-5,6-diphenyl-1,2,4-triazines [6] and 3-amino1,2,4-triazines [7]. In addition, functionalized bridgehead nitrogen heteroannulated 1,2,4-triazine systems works as pharmacological probes [10] while the pyrimidine nucleus and their derivatives exhibit a wide range of pharmacological, medicinal and biological properties [11].

Consequently, this investigation tends to synthesis barbituric and thiobarbituric acids as pyrimidine nucleus bearing a bioactive 5,6-diphenyl-1,2,4-triazin-3-yl moiety and their evaluate as CDK2 inhibitors of tumor cells.

\section{Chemistry}

3-Amino-5,6-diphenyl-1,2,4-triazine (1) [7] as starting material, is obtained by cyclocondensation of benzyl with aminoguanidine bicarbonate in reflux n-butanol. The Addition of cyclohexyl isocyanate, methyl isothiocyanate, and phenyl isothiocyante to compound 1 in reflux EtOH, produced $N^{l}, N^{3}$-disubstituted urea 2 and $N^{l}, N^{3}$-disubstituted 
thioureas 3 and 4 respectively (Figure 1).

To deduced the aim of this work, the compounds 2-4 on ring closure reactions with malonic acid in refluxing glacial acetic acid afforded the $N^{l}$-(cyclohexyl)- $N^{3}-\left(5^{\prime}, 6^{\prime}\right.$-diphenyl- 1',2',4'-triazin-3'-yl)barbituric acid (5) and/or $\quad \mathrm{N}^{1}$ (methyl/phenyl)- $\mathrm{N}^{3}-\left(5^{\prime}, 6^{\prime}\right.$-diphenyl-1,2,4-triazin-3'-yl) thiobarbituric acids (6 and 7) respectively [12], Figure 1.

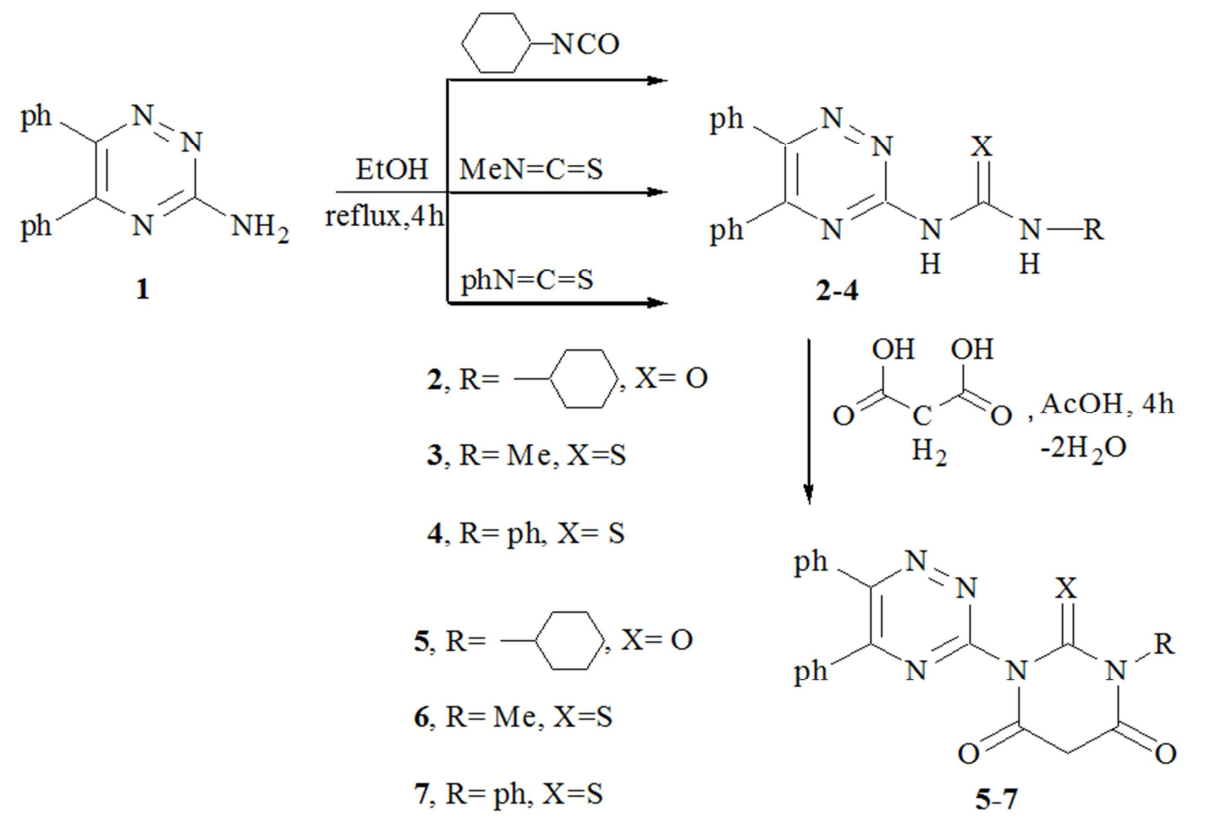

Figure 1. Ring closure reactions on compounds 2-4 in to give barbituric acid (5) and thiobarbituric acids (6 and 7).

The active methylene [13] in the skeleton of compound 57 at C-5 can be confirmed by condensation with pyridine-4carboxyladehyde to give 1-(cyclohexyl/methyl/phenyl)-3(5,6-diphenyl-1',2',4'-triazin-3'-yl)-5-(pyridi-4-ene)barbituric and thiobarbituric acids (8-10) (Figure 2).

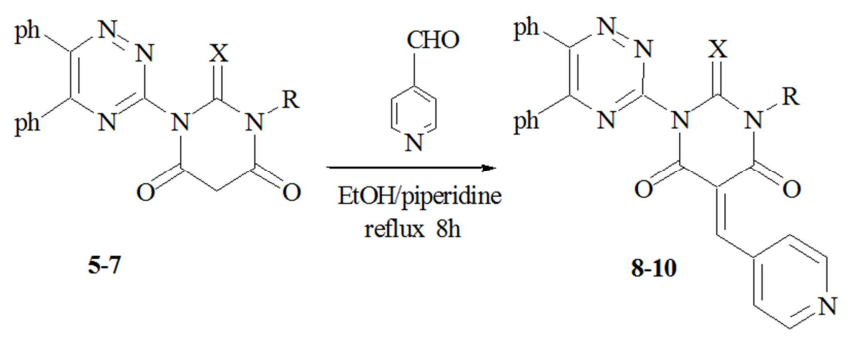

Figure 2. Synthesis of thiobarbituric acids (8-10).

The introducing of fluorine atoms to heterocyclic nitrogen systems, often improve and enhance the physical, chemical and medicinal properties [3, 4] Thus, fluoroacylation of compounds $5-7$ by refluxing with hexafluoroacetic anhydride in THF, afforded 1-(cyclohexyl/methyl/phenyl)-3-(5',6'diphenyl-1',2',4'-triazin-3'-yl)-5-(trifluoracetyl)-5Hbarbituric/thiobarbituric acids (11-13) (Figure 3).<smiles>[X]C1N([R])C(=O)CC(=O)N1c1nnc([TlH])c(P)n1</smiles>

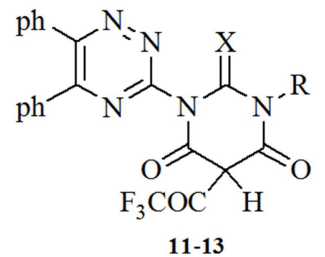

Figure 3. Synthesis of barbituric/thiobarbituric acids (11-13).

\section{Results and Discussion}

This work reports a simple synthesis strategy toward both 5,6-diphenyl-3-amino-1,2,4-triazine (1) and the substituted barbituric/thiobarbituric acids, Structures of the new targets obtained established from their correct elemental analysis and spectral measurements.

Thus, IR spectra of 1,3-disubstituted urea/thiourea (2 and 3) showed peak at $3180,1630,1385,1195 \mathrm{~cm}^{-1}$ attribute to $\mathrm{NH}, \mathrm{CO}$, acyclic $\mathrm{NCSN}$, and $\mathrm{C}=\mathrm{S}$ functional groups. While compound give peaks at $1190(\mathrm{C}=\mathrm{S}), 3160(\mathrm{NH})$, and 1330 $\mathrm{cm}^{-1}$ (acyclic NCSN).

Structures of both substituted barbituric acids and thiobarbituric acids (5-7) can be deduced from spectral data. the UV spectrum of 7 recorded $\lambda_{\max }$ at 260 (nm, $\left.\varepsilon, 0.95\right)$, while that IR showed peaks at $3470(\mathrm{OH}), 1680(\mathrm{C}=\mathrm{O}), 1441$ (deformation of aliphatic $\mathrm{CH}_{2}$ ), 1387 (cyclic NCSN), 1580, $1560(\mathrm{C}=\mathrm{N}), 1180(\mathrm{C}=\mathrm{S}), 860$ and $810 \mathrm{~cm}^{-1}$ (phenyl). The NMR spectrum of 7 showed a the hydroxy resonated proton as signals at $\delta 9.8$, complicated aromatic protons at 7.967.77, 7.6-7.2 and active methylene two protons at 2.59-2.56 acids. Additionally, Mass fragmentation of compound 7 showed the molecular ion at $\mathrm{m} / \mathrm{e} 451$ (1.01) with the base peak at m/e 178 (100) attribute to diphenylacetylene radical [14].

According to the condensation of compound 6 with pyridin-4-carboxaldehyde the active methyl group (C-5) not showed as expected in the IR, ${ }^{1} \mathrm{H}$ NMR and ${ }^{13} \mathrm{C}$ NMR spectra of compounds 8-10, ppm. Furthermore, IR spectrum showed only two carbonyl groups at $v 1720,1700 \mathrm{~cm}^{-1}$ and $(\mathrm{C}=\mathrm{S})$ at $1188 \mathrm{~cm}^{-1}$ 
On the other hand, fluoroacetylation of compound 6 by reflux with hexafluoroacetic anhydride in THF afforded the mono (trifluoroacetyl) derivative 12 . Structure of compound 12 confirmed by rang of spectrum analysis, IR spectrum showed broad hydroxyl peak of $(\mathrm{OH})$ at 3450-3400, carboxylic $\mathrm{C}=\mathrm{O}$ at $1700 \mathrm{~cm}^{-1}$, while aliphatic $\mathrm{CH}$ at $\mathrm{C}-5$ appears at $1414 \mathrm{~cm}^{-1}$. thiobarbituric acid $(\mathrm{C}=\mathrm{S})$ at position-3 showed peaks at $1180 \mathrm{~cm}^{-1} \cdot{ }^{13} \mathrm{C}$ NMR of 12 recorded the presence of resonated carbon signals at $\delta 180,166,146,142$, $138,132-122$ and $40 \mathrm{ppm}$ for $\mathrm{C}=\mathrm{S}, \mathrm{C}=\mathrm{O}, \mathrm{C}-\mathrm{F}, \mathrm{C}=\mathrm{N}, \mathrm{C}=\mathrm{C}$, $\mathrm{CH}_{3}$ respectively, in addition to aromatic carbons of 1,2,4triazine moiety.

Mass fragmentation of compound 12 showed m/e at lower percentage, which may be a less stability of the fluorinated systems, with a base peak at $m / e 85$ attribute to $\mathrm{COCF}_{3}$ ions.

\section{Experimental}

Melting points were determined with an electrochemical Bibly Sturat Scientific melting point sample (UK). A Perkin Elmer Model RXI-FT IR system 55529 was used for Recording IR spectra of the prepared compounds $\left(\mathrm{cm}^{-1}\right)$. the ${ }^{1} \mathrm{H}$ and ${ }^{13} \mathrm{CNMR}$ spectra DMSO-d $\mathrm{d}_{6}(\mathrm{ppm})$, A Bruker advance DPX 400 MHZ model uses TMS an internal standard. A GCMS-GP 1000 Ex model was used for recording the mass spectra of the compounds $(\mathrm{MHz})$. Electronic spectra were recorded in ethanol on Shimadzu UV and visible 310 IPC Spectrophotometer $(\mathrm{nm})$. Elemental analysis was performed in micro analytical Center of Cairo University, Cairo, Egypt. 3-Amino-5,6-diphenyl-1,2,4-triazine (1) prepared according the reported method [7].

\subsection{Synthesis of 1-(Cyclohexyl)-3-(5',6'-diphenyl-1,2,4- triazin-3'-yl) Urea (2)}

A mixture of compound $1(0.01 \mathrm{~mol})$ and cyclohexyl isocyanate $(0.01 \mathrm{~mol})$ in abs. EtOH $(50 \mathrm{ml})$ refluxed $2 \mathrm{~h}$, cooled. The solid was collected and crystallized from EtOH to give 2. Yield\%; m.p. $167-168^{\circ} \mathrm{C}$. IR $(v) \mathrm{cm}^{-1}: 3180(\mathrm{NH})$, 2980, 2888 (aliphatic $\mathrm{CH}_{2}$ ), 1630-1620 (CONH), 1580 $(\mathrm{C}=\mathrm{N}), 1487,1441$ (bending $\mathrm{CH}), 870,850,810$ (phenyls). ${ }^{1} \mathrm{H}$ NMR (DMSO-d 6 ) $\delta$ ppm: $10.08(s, 1 \mathrm{H}, \mathrm{b}, \mathrm{NH}), 9.15(s$, $1 \mathrm{H}, \mathrm{b}, \mathrm{NH}), 7.7-7.2(\mathrm{~m}, 10 \mathrm{H}$, aromatic); $2.15(\mathrm{~s}, 1 \mathrm{H}, \mathrm{CH})$, 1.55, 1.50 (each m, 4- $\left.\mathrm{CH}_{2}\right), 0.8\left(s, 3 \mathrm{H}, \mathrm{CH}_{3}\right)$. Anal. Calcd.; C, 70.77; H, 6.16; N, 18.76\% for $\mathrm{C}_{22} \mathrm{H}_{23} \mathrm{~N}_{5} \mathrm{O}$ (373). Found: C, $70.73 ; \mathrm{H}, 6.16 ; \mathrm{N}, 18.62 \%$.

\subsection{Synthesis of 1-(Methyl/phenyl)-3-(5',6'-diphenyl- 1',2',4'-triazin-3'-yl) Thioureas (3 and 4)}

A mixture of $1(0.01 \mathrm{~mol})$, methyl isothiocyanate and/or phenyl isothiocyanate $(0.01 \mathrm{~mol})$ in abs. EtOH $(50 \mathrm{ml})$ refluxed $2 \mathrm{~h}$, cooled. The produced solid filtered off and crystallized from EtOH, to give 3 and 4 respectively.

3, Yield 79\%; m.p. 180-182 ${ }^{\circ}$ C., Anal. Calcd.; C, 63.55; H, 4.67; N, 21.80; S, 9.96\% for $\mathrm{C}_{17} \mathrm{H}_{15} \mathrm{~N}_{5} \mathrm{~S}$ (321). Found: $\mathrm{C}$, 63.42; H,4.58; N,21.74; S, 10.01 .

4, Yield $81 \%$; m.p. $188-190^{\circ} \mathrm{C}$. UV (EtOH) $\lambda_{\max } 240 \mathrm{~nm}$,
IR (v) $\mathrm{cm}^{-1}: 2880\left(\mathrm{CH}_{2}\right), 1610(\mathrm{C}=\mathrm{C}), 1580(\mathrm{C}=\mathrm{N}), 1350$ (acyclic NCSN), $1190(\mathrm{C}=\mathrm{S}), 850,810,790$ (phenyls). ${ }^{1} \mathrm{H}$ NMR (DMSO-d $\left.{ }_{6}\right) \delta$ ppm: $10.34(1 \mathrm{H}, \mathrm{b}, \mathrm{NH}), 9.34(1 \mathrm{H}, \mathrm{b}$, $\mathrm{NH})$, 7.67-7.2 (m, 10H, aromatic), 7.0-6.88 (m, 5H, phenyl protons). Anal. Calcd.; C, 68.92; H, 4.43; N, 18.27; S, 8.35\% for $\mathrm{C}_{22} \mathrm{H}_{17} \mathrm{~N}_{5} \mathrm{~S}$ (383). Found: C, 68.86; H, 4.37; N, 18.07; S, $8.29 \%$.

\subsection{1-(Cyclohexyl)-3-(5',6'-diphenyl-1',2',4'-triazin-3'-yl) Barbituric Acid (5)}

Equimolar amounts of 2 and malonic acid in glacial $\mathrm{AcOH}$ $(50 \mathrm{ml})$ refluxed for $4 \mathrm{~h}$, cooled then concentrated. The solid produced, filtered off and crystallized from dioxin to give 5, yield $65 \%$, m.p. $180-182^{\circ} \mathrm{C}$. IR $(v) \mathrm{cm}^{-1}: 3470(\mathrm{OH}), 1700-$ $1680(2 \mathrm{C}=\mathrm{O}), 1480,1441$ (bending $\mathrm{CH}), 850,810$ (phenyls). ${ }^{1} \mathrm{H}$ NMR (DMSO-d $\left.{ }_{6}\right) \delta$ ppm: 7.7-7.44 (m, 10H, aromatic), $3.52(s, 1 \mathrm{H}$, enolic HO-C"H), $2.59(s, 1 \mathrm{H}, \mathrm{CH}=\mathrm{C}), 1.20(\mathrm{~s}$, $\left.3 \mathrm{H}, \mathrm{CH}_{3}\right) .{ }^{13} \mathrm{C}$ NMR (DMSO-d 6 ) $\delta$ ppm: $165.65,159.63$ and $158.02(3 \mathrm{C}=\mathrm{O}), 134.52(\mathrm{C}=\mathrm{N}), 132-122$ (aromatic carbons), $40\left(\mathrm{CH}_{3}\right)$. Anal. Calcd.; C, 68.02; H, 5.21; N, 15.87\% for $\mathrm{C}_{25} \mathrm{H}_{23} \mathrm{~N}_{5} \mathrm{O}_{3}$ (441). Found: C, 68.12; H, 5.18; N, 15.90 .

\subsection{1-(Methyl/phenyl)-3-(5',6'-diphenyl-1,2,4-triazin-3'-yl) Thiobarbituric Acids (6 and 7)}

A mixture of 3 and/or $4(0.01 \mathrm{~mol})$ and malonic acid $(0.01$ mol) in glacial AcOH $(50 \mathrm{ml})$ refluxed $4 \mathrm{~h}$, cooled then concentrated. The solid obtained filtered off and crystallized from dioxin to give 6 and 7 respectively.

6: Yield 73\%, m.p. $203-205^{\circ}$ C. Anal. Calcd.; C, 61.69; H, 3.85; N, 17.99 for $\mathrm{C}_{20} \mathrm{H}_{15} \mathrm{~N}_{5} \mathrm{SO}_{2}$ (389). Found: C, 61.73; H, $3.81 ; \mathrm{N}, 18.09 \%$. [15]

7: Yield\%, m.p. $193-195^{\circ} \mathrm{C}$. UV (EtOH) $\lambda_{\max }: 260 \mathrm{~nm}(\varepsilon$, 0.95). IR (v) $\mathrm{cm}^{-1}: 3470(\mathrm{OH}), 1710,1680(2 \mathrm{C}=\mathrm{O}), 1610$ $(\mathrm{C}=\mathrm{C}), 1580(\mathrm{C}=\mathrm{N}), 1488,1440$ (bending $\mathrm{CH}), 1330$ (cyclic NCSN), $1180(\mathrm{C}=\mathrm{S}), 880,860,810,780$ (phenyls). ${ }^{1} \mathrm{H}$ NMR $\left(\right.$ DMSO-d $\left._{6}\right) \delta$ ppm: 7.96, 7.77, 7.6-7.2; 7.01-6.8 (each m, $15 \mathrm{H}$, aromatic), $9.8\left(s, 1 \mathrm{H}\right.$, enolic $\left.\mathrm{OH}-\mathrm{C}^{\prime \prime}\right), 2.59(s, \mathrm{CH}=\mathrm{C})$. ${ }^{13} \mathrm{C}$ NMR (DMSO-d 6 ) $\delta$ ppm: $180.1(\mathrm{C}=\mathrm{S}), 165,159(\mathrm{C}=\mathrm{O})$, 142, $135(\mathrm{C}=\mathrm{N}), 128-122$ (aromatic carbons). $\mathrm{M} / \mathrm{S}$ (Int.\%): $451\left(\mathrm{M}^{+}, 1.01\right)$; 178 (100), 135 (15), 84 (5.71), 70 (85.13), 56 (5.18). Anal. Calcd.; C, 66.51; H, 3.76; N, 15.52\% for $\mathrm{C}_{25} \mathrm{H}_{17} \mathrm{~N}_{5} \mathrm{SO}_{2}$ (451). Found: C, 66.56; H, 3.71; N, 15.48;\%.

\subsection{1-(Cyclohexyl)-3-(5',6'-diphenyl-1',2',4'-triazin-3'-yl)- 5-(pyrid-4-ene) Barbituric Acid (8)}

Equimolar amounts of 5 and pyridine-4-carboxaldehyde in EtOH $(100 \mathrm{ml})$ with few drops of piperidine refluxed $8 \mathrm{~h}$, cooled, then poured onto ice-drops $\mathrm{AcOH}$. The solid yielded filtered off and crystallized from dioxin to give 8, Yield 78\%; m.p. $195-197^{\circ} \mathrm{C}$. IR (v) $\mathrm{cm}^{-1}: 3050$ (aromatic CH), 1710, 1680, $1660(3 \mathrm{C}=\mathrm{O}), 1580(\mathrm{C}=\mathrm{N}), 1488,1441$ (bending $\mathrm{CH})$, 850, 810 (phenyls). ${ }^{1} \mathrm{H}$ NMR (DMSO-d 6 ) $\delta$ ppm: $9.23(s$, arylidene proton), 8.2, 8.0 (each dd, 2CH-pyridine), 7.8-7.66, 7.40-7.2, 7.0-6.69 (m, 18H, aromatic and pyridine). ${ }^{13} \mathrm{C} \mathrm{NMR}$ $\left(\mathrm{DMSO}_{\mathrm{d}}\right) \delta$ ppm: 165.67, $160.91(\mathrm{C}=\mathrm{O}), 138.4(\mathrm{C}=\mathrm{N}), 133$ $(\mathrm{C}=\mathrm{C}), \quad 130-122$ (aromatic carbons), 40, 24 (aliphatic 
carbons). Anal. Calcd.; C, 68.91; H, 4.96; N, 16.63\% for $\mathrm{C}_{29} \mathrm{H}_{25} \mathrm{~N}_{6} \mathrm{O}_{3}$ (505). Found: $\mathrm{C}, 67.88 ; \mathrm{H}, 4.80 ; \mathrm{N}, 15.59$.

\subsection{1-(Methyl/phenyl)-3-(5',6'-diphenyl-1',2',4'-triazin-3'- yl)-5-(pyridi-4-ene)-Thiobarbituric Acids (9 and 10)}

A mixture of 6 and $7(0.01 \mathrm{~mol})$ and pyridine-4carboxaldehyde $(0.01 \mathrm{~mol})$ in $\mathrm{EtOH}(100 \mathrm{ml})$ and $2 \mathrm{ml}$ of piperidine, refluxed for $8 \mathrm{~h}$, after cooled. The reaction mixture poured onto cold water $50 \mathrm{ml}$ with $1 \mathrm{ml}$ of $\mathrm{AcOH}$. Collect the obtained solid by filtration and recrystallized from $\mathrm{EtOH}$ to give 9 and 10 respectively.

9, Yield 78\%; m.p. $168-170^{\circ} \mathrm{C}$. Anal. Calcd.; C, 64.51; H, $3.65 ; \mathrm{N}, 18.06 ; \mathrm{S}, 6.88 \%$ for $\mathrm{C}_{25} \mathrm{H}_{17} \mathrm{~N}_{6} \mathrm{SO}_{2}$ (465). Found: $\mathrm{C}$, 64.47; H, 3.45; N, 17.97; S, 6.83\%.

10, Yield $61 \%$; m.p. $180-182^{\circ} \mathrm{C}$. IR (v) $\mathrm{cm}^{-1}: 3060$ (aromatic CH), 1720, $1700(\mathrm{C}=\mathrm{O}), 1610(\mathrm{C}=\mathrm{C}), 1590,1560$ $(\mathrm{C}=\mathrm{N}), 1330$ (cyclic NCS), $1170(\mathrm{C}=\mathrm{S}), 910,850,810$ (phenyls). ${ }^{1} \mathrm{H}$ NMR (DMSO-d 6 ) $\delta$ ppm: $7.85(s, 1 \mathrm{H}, \mathrm{CH}=$ pyridine), 7.6, 7.7 (each d, d, 2CH-pyridine), 7.6-6.9, 6.76.55 (each $\mathrm{m}, 18 \mathrm{H}$, aromatic \& pyridine). ${ }^{13} \mathrm{C}$ NMR (DMSO$\left.\mathrm{d}_{6}\right) \delta$ ppm: $181(\mathrm{C}=\mathrm{S}), 165,160(\mathrm{C}=\mathrm{O}), 142(\mathrm{C}=\mathrm{N}), 134.4$ $(\mathrm{CH}=\mathrm{C}$ arylidene), 132-122 (aromatic \& pyridine carbons).

Anal. Calcd.; C, 68.31; H, 3.60; N, 15.93; S, 6.07\% for $\mathrm{C}_{30} \mathrm{H}_{19} \mathrm{~N}_{6} \mathrm{SO}_{2}$ (527). Found: C, 68.28; H, 3.64; N, 15.88; S, $6.11 \%$.

\subsection{1-(Cyclohexyl)-3-(5',6'-diphenyl-1',2',4'-triazin-3'-yl)- 5-(trifluoroacetyl) Barbituric Acid (11)}

A mixture of $5(0.01 \mathrm{~mol})$ and hexafluoroacetic anhydride $(0.015 \mathrm{~mol})$ in THF $(100 \mathrm{ml})$ refluxed $2 \mathrm{~h}$, cooled. The resulted solid was collected by filtration and crystallized from dioxin to give 11 , yield $71 \%$; m.p. $168-170^{\circ} \mathrm{C}$. IR $(v)$ $\mathrm{cm}^{-1}$ : 3060 (aromatic CH), 1720, 1700, $1670(\mathrm{C}=\mathrm{O}), 1610$ $(\mathrm{C}=\mathrm{C}), 1580(\mathrm{C}=\mathrm{N}), 1480,1441$ (bending $\left.\mathrm{CH}_{2}\right), 1230(\mathrm{C}-\mathrm{F})$, 910, 880, 810 (phenyls), 750 (C-F).

${ }^{1} \mathrm{H}$ NMR (DMSO-d $\left.{ }_{6}\right) \delta$ ppm: $8.51(s, 1 \mathrm{H}, \mathrm{OH}), 7.6-7.4$, 7.2-6.95 (m, 10H, aromatic), $2.58(s, \mathrm{CH}), 1.5,1.25,1.20$ (each $s, 3 \mathrm{CH}_{2}$ ). ${ }^{13} \mathrm{C}$ NMR (DMSO-d 6 ) $\delta$ ppm: $167.52,166.37$ $(2 \mathrm{C}=\mathrm{O}), 151(\mathrm{C}-\mathrm{OH}), 146(\mathrm{C}-\mathrm{F}), 142(\mathrm{C}=\mathrm{N}), 132-127$ (aromatic carbons), 40.44, 39.54, 25 (aliphatic carbons). Anal. Calcd.; C, 60.33; H, 4.09; N, 13.03; F, 10.61\% for $\mathrm{C}_{27} \mathrm{H}_{22} \mathrm{~N}_{5} \mathrm{~F}_{3} \mathrm{O}_{4}$ (537). Found: $\mathrm{C}, 60.03 ; \mathrm{H}, 4.12 ; \mathrm{N}, 12.96 ; \mathrm{F}$, $10.65 \%$.

\subsection{1-(Methyl/phenyl)-3-(5',6'-diphenyl-1',2',4'-triazin-3'- yl)-5-(trifluoroacetyl) Thiobarbituric Acids (12 and 13)}

A mixture of 6 or $7(0.01 \mathrm{~mol})$ and hexafluoroacetic anhydride $(0.01 \mathrm{~mol})$ in THF $(100 \mathrm{ml})$ reflux for $2 \mathrm{~h}$, cooled. The solid obtained, filtered off and crystallized from dioxin to give 12 and 13 respectively.

12, Yield62 \%; m.p. $158-160^{\circ} \mathrm{C}$. IR (v) $\mathrm{cm}^{-1}: 3450(\mathrm{OH})$, 3060 (aromatic CH), 2980, 2880 (aliphatic $\mathrm{CH}), 1700(\mathrm{C}=\mathrm{O})$, $1680(\mathrm{C}=\mathrm{O}), 1610(\mathrm{C}=\mathrm{C}), 1590(\mathrm{C}=\mathrm{N}), 1350($ cyclic $\mathrm{CH})$, 1240 (C-F), $1188(\mathrm{C}=\mathrm{S}), 910,850,810$ (phenyls), 780 (C-F). ${ }^{1} \mathrm{H}$ NMR (DMSO-d $\left.{ }_{6}\right) \delta$ ppm: $8.8(s, 1 \mathrm{H}, \mathrm{CH}=$ cyclic), 7.87.66, 7.4-6.95 (each $\mathrm{m}, 10 \mathrm{H}$, aromatic), $1.25\left(s, 3 \mathrm{H}, \mathrm{CH}_{3}\right)$.
${ }^{13} \mathrm{C}$ NMR (DMSO-d 6 ) $\delta$ ppm: $180(\mathrm{C}=\mathrm{S}), 166(\mathrm{C}=\mathrm{O}), 146$ (C-F), $142(\mathrm{C}=\mathrm{N}), 138(\mathrm{C}-\mathrm{N}), 132-122$ (aromatic carbons), $40\left(\mathrm{CH}_{3}\right.$ carbons). $\mathrm{m} / \mathrm{s}$ (Int.\%): 485 (487, $\left.\mathrm{M}+2,5.03\right): 178$ (85.11), 113 (75.11), 85 (100), 69 (5.13). Anal. Calcd.; C, $54.43 ; \mathrm{H}, 2.88 ; \mathrm{N}, 14.43 ; \mathrm{F}, 11.7 ; \mathrm{S}, 6.58 \%$ for $\mathrm{C}_{22} \mathrm{H}_{14} \mathrm{~N}_{5} \mathrm{~F}_{3} \mathrm{SO}_{3}$ (485). Found: C, 54.38; H, 2.88; N, 14.45; F, 11.67; S, 6.54\%

13, Yield 59\%; m.p. $178-180^{\circ} \mathrm{C}$. Anal. Calcd.; C, 59.23; H, 2.92; N, 12.79; F, 10.42; S, 8.5\% for $\mathrm{C}_{27} \mathrm{H}_{16} \mathrm{~N}_{5} \mathrm{~F}_{3} \mathrm{SO}_{3}$ (547). Found: C, 59.03; H, 2.96; N, 12.82; F, 10.37; S, 8.53\%.

\section{Inhibition of Cyclin-Dependent Kinase 2 (CDK2) for Cell Tumor Division}

The purine-related pyrrolo[2,1-f][1,2,4]triazines have been identified as tyrosine kinase inhibitors, a well-established platform for modern anticancer chemotherapy [15]. Recent control on the cell tumor division depends on use of polyfunctional heterocyclic nitrogen systems as CDK2 for examples fluorine substituted thiobarbituric acid [16].

Recently, number of CDK inhibitors have been developed. Some compounds of the series were able to inhibit activity of CDK2 in a biochemical assay with IC50 values comparable to olomoucine [17]. The most active compounds with trifluoroacetyl at position 5, such as 5(trifluoroacetyl)barbituric acid (11) and 5(trifluoroacetyl)thiobarbituric acids (12 and 13) In addition we have observed that a pyredine side chain at C-5 significantly decreases CDK2 inhibitory activity (5-(pyridi-4ene)-thiobarbituric acids compound 9 and 10):). The data on CDK2 inhibition of $[1,2,4]$ triazines derivatives summarized in (Table 1).

Generally, this investigation showed that Thiobarbituric acids 6 and 7 showed activity over than barbituric acid 5 and their thiourea derivatives 3 and 4 . While trifluoroacetylthiobarbituric acid 12 Record higher activity over the corresponding thiobarbituric acid 6. Furthermore, compound 12 had a higher percentage of fluorine elements, which led to a higher hydrophobic, distribution, and H-bonding formation. Table 1.

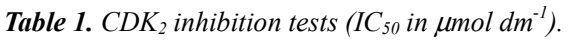

\begin{tabular}{ll}
\hline Compound. No. & IC $_{\mathbf{5 0}} \mathbf{C D K}_{\mathbf{2}} \pm \mathbf{S D}(\boldsymbol{\mu M o l})$ \\
\hline 2 & $>20$ \\
3 & $14.1 \pm 1.9$ \\
4 & $>20$ \\
5 & $11.2 \pm 5.3$ \\
6 & $5.50 \pm 4.5$ \\
7 & $10.15 \pm 3.8$ \\
8 & $15.2 \pm 1.7$ \\
9 & $16.1 \pm 1.8$ \\
10 & $18.5 \pm 2.8$ \\
11 & $4.8 \pm 1.7$ \\
12 & $4.2 \pm 1.0$ \\
13 & $4.5 \pm 1.0$ \\
Olomoucine & $5.0 \pm 1.0$ \\
\hline
\end{tabular}

SD: standard deviation

Olomoucine value is included as control [17]. 


\section{Conclusion}

Fluorinated thiobarbituric and barbituric acids and their related systems, have been synthesis by simple effective methodology by the addition of isocyanate and isothiocyanate to 3-amino-1,2,4-triazine followed by ring closure reactions with malonic acid and finally condensation with fluoroacetylation. The novel synthesized systems is preliminary evaluated as CDK2 for cell tumor division, compounds with sulfur and trifluoroacetyl exhibit a higher activity over standard and other synthesis compounds.

\section{References}

[1] C. Schiffer, (2007). BCR-ABL tyrosine kinase inhibitors for chronic myeloge-nous leukemia. N. Engl. J. Med. 357, 258265.

[2] P. Cohen, (2002). Protein kinases - the major drug targets of the twenty-firstcentury? Nat. Rev. Drug Discov. 1, 309-315.

[3] V. Miduturu, X. Deng, N. Kwiatkowski, W. Yang, L. Brault, P. Filippakopoulos, E. Chung, Q. Yang, J. Schwaller, S. Knapp, R. King, J. Lee, S. Herrgard, P. Zarrinkar, N. Gray, (2011). High-Throughput Kinase Profiling: A More Efficient Approach toward the Discovery of New Kinase Inhibitors, $\begin{array}{llll}\text { Chemistry \& Biology, } 18 \text { (7), 868-879, } & \text {, }\end{array}$ doi.org/10.1016/j.chembiol.2011.05.010.

[4] B. Mathew, J. Suresh, D. Vinod., (2012). Antitumor activity of 5-[(2E)-1-(1H-benzimidazol-2-yl)-3-substituted-phenylprop-2en-1-ylidene]pyrimidine2,4,6(1H,3H,5H)-triones against Dalton's ascetic lymphoma in mice. Med Chem Res 22: 39113917.

[5] T. Venkatesh, Y. Bodke, R. Kenchappa and S. Venkatesh, (2016). Synthesis, Antimicrobial and Antioxidant Activity of Chalcone Derivatives Containing Thiobarbitone Nucleus. Med chem (Los Angeles), 6 (7), 440-448. DOI: 10.4172/21610444.1000383 .

[6] D. Bakhotmah, R. Abdel-Rahman, (2016), A Review on the Synthesis and Chemistry of Bioactive Pyrazolines Bearing 1,2,4-Triazine Moieties, Mini-Reviews in Organic Chemistry, 13, 62-77. 13 (1): 62-77.

[7] R. Abdel-Rahman, M. Makki, T. Ali, M. Ibrahim, (2015), 1,2,4-Triazine chemistry Part IV: Synthesis and chemical behavior of 3-functionalized 5,6-diphenyl-1,2,4-triazines towards some nucleophilic and electrophilic reagents, J. Heterocyclic chem. 52: 1595-1607.
[8] A. Bazgir, M. Khanaposhtani and A. Soorki, (2008), One-pot synthesis and antibacterial activities of pyrazolo[4',3':5,6]pyrido[2,3-d]pyrimidine-dione derivatives. Bioorganic and medicinal chemistry letters, 18, 5800-5803.

[9] M. Masoud, M. Awad, M. Shaker, M. El-Tahawy, (2010), The role of structural Chemistry in the inhibitive performance of some aminopyrimidines on the corrosion of steel, Corrosion Science, 52 (7), 2387-96.

[10] R. Abdel-Rahman, M. Makki, M. Ali, M. Ibrahim, (2013), 1,2,4-Triazine Chemistry Part III: Synthetic Strategies to Functionalized Bridgehead Nitrogen Heteroannulated 1,2,4Triazine Systems and Their Regiospecific and Pharmacological Properties. Current Organic Chemistry, 10, 136-160.

[11] R. Abdel-Rahman, K. El-mahdy, (2012), Biological Evaluation of Pyramidpyrimidines as Multi-Targeted Small Molecule Inhibitors and Resistance Modifying Agents. Heterocycles, 85, 2391-2414. doi.org/10.3987/REV-12-745.

[12] B. Vinosha, S. Perumal, S. Renuga, A. Almansour, (2012) A facile domino protocol for the stereoselective synthesis of trans-2,3-dihydrobenzofurans and cis-5, 6-dihydrofuro[2,3d]pyrimidines, Tetrahedron Letters, 53, 962-966.

[13] L. Ma, S. Li, H. Zheng, J. Chen, L. Lin, X. Ye, Z. Chen, Q. Xu, T. Chen and J. Yang, (2011), Synthesis and biological activity of novel barbituric and thiobarbituric acid derivatives against non-alcoholic fatty liver disease. European journal of medicinal chemistry, 46 (6): 2003-10. doi: 10.1016/j.ejmech.2011.02.033.

[14] M. Palmer, P. Preston and M. F. Stevens, The Mass Spectra of 1,2,4-Triazines and Related Compounds. Organic Mass Spectrometry Organic Mass Spectrometry 1971, 5, 1085-1092.

[15] J. Hunt, T. Mitt, R. Borzilleri, J. Gullo-Brown, J. Fargnoli, B. Fink, W. C Han, S. Mortillo, G. Vite, B. Wautlet, T. Wong, C. Yu, X. Zheng, R. Bhide, (2004) Discovery of the pyrrolo[2,1f] $[1,2,4]$ triazine nucleus as a new kinase inhibitor template. J Med Chem 47 (16): 4054-9.

[16] T. Gucky, E. Řezníčková, P. Dzubak, Hajduch, K. Marian, (2010). ChemInform Abstract: Synthesis and Anticancer Activity of Some 1,5-Diaryl-3-(3,4,5-trihydroxyphenyl)-1Hpyrazolo[4,3-e][1,2,4]triazines, Monatsh Chem., 141: 709-714. 10.1007/s00706-010-0314-4.

[17] A. Senderowicz, Inhibitors of cyclin-dependent kinase modulators for cancer therapyProgress in Drug Research (2005), Progress in Drug Research. 63, 183-206. 